\title{
Ferrite Characterization for the Design of an Accelerating Cavity With Perpendicular Biasing
}

\author{
J. Eberhardt, F. Caspers, Senior Member, IEEE, and C. Vollinger, Member, IEEE
}

\begin{abstract}
A perpendicular biased ferrite loaded accelerating cavity is studied for a possible upgrade of the CERN accelerator complex which could help to overcome the intensity limitations occurring at the SPS injection. The required accelerating cavity should cover a frequency range of 18 to $40 \mathrm{MHz}$ with high cavity $Q$, which poses high demands on the ferrite material properties. A test setup is presented to measure the relative permeability and magnetic losses of full scale ferrite garnets $(350 \mathrm{~mm}$ outer and $200 \mathrm{~mm}$ inner diameter) in a magnetic bias field within the frequency range of interest. An one-port reflection measurement provides adequate input to model the relative permeability of the ferrite in numerical simulations for different magnetic bias fields. A resonant measurement setup was used to cross-check simulation results with measurement data and to investigate the magnetic losses of the ferrite material. A numerical model of a simplified accelerating cavity is used to study the capability of the garnet G-510 as a perpendicular biased tuning ferrite.
\end{abstract}

Index Terms-Ferrite loaded accelerating cavity, ferrites, garnets, permeability measurement, reflection measurement, resonant measurement.

\section{INTRODUCTION}

$\mathbf{T}$ HE resonance frequency of a ferrite loaded accelerating cavity shifts by applying an external magnetic bias field to the ferrite material by means of changing the relative permeability. For the design process of such cavities, it is essential to know the electromagnetic properties of the ferrite material. The complex relative permeability and permittivity can be expressed as: $\mu_{\mathrm{r}}=\mu^{\prime}-i \mu^{\prime \prime}$ and $\varepsilon_{\mathrm{r}}=\varepsilon^{\prime}-i \varepsilon^{\prime \prime}$, respectively. Often, only little information about the electromagnetic material properties is available in the frequency range of interest. Hence, the material has to be characterized by appropriate measurements.

Conventional ferrite loaded accelerating cavities make use of a magnetic bias field which is oriented in parallel to the RF magnetic field. It was shown by Earley that a magnetic bias field which is perpendicular to the RF magnetic field can lead to a much higher magnetic $Q$ of the ferrite in comparison to parallel biasing [1]. Prototypes of such cavities were built and tested e.g. for the SSC [2] and at TRIUMF [3]. To our knowledge,

Manuscript received May 29, 2015; revised July 08, 2015; accepted July 24, 2015. Date of publication October 30, 2015; date of current version April 15, 2016.

J. Eberhardt is with the European Organization for Nuclear Research (CERN), CH-1211 Geneva 23, Switzerland, and also with Technische Universitaet Darmstadt, Institut fuer Theorie Elektromagnetischer Felder, D-64289 Darmstadt, Germany (e-mail: johannes.eberhardt@cern.ch).

F. Caspers and C. Vollinger are with the European Organization for Nuclear Research (CERN), CH-1211 Geneva 23, Switzerland.

Color versions of one or more of the figures in this paper are available online at http://ieeexplore.ieee.org.

Digital Object Identifier 10.1109/TNS.2015.2463091 no perpendicular biased ferrite loaded accelerating cavity is in operation at the moment. Except our research, design studies of perpendicular biased ferrite tuned cavities are currently performed for the Fermilab booster update in [4] and [5]. Within our project, a series of measurements using different measurement methods, magnetic bias field orientations and various ferrite sample sizes were conducted recently. First, different types of ferrite tiles have been evaluated [6] for use in an accelerating cavity with a frequency sweep of 18 to $40 \mathrm{MHz}$. A measurement technique was presented to calculate the relative permeability vs. frequency, dependent on a static magnetic bias field of different orientations. The garnet G-510 from Trans-Tech, Inc. [7] was evaluated to be most suitable for perpendicular biasing within a frequency range of 18 to $40 \mathrm{MHz}$ with regards to the available range in relative permeability and related magnetic losses. In [8] and [9] a possible resonance sweep was investigated by using G-510 toroidal samples in a re-entrant measurement cavity. It was shown that superposition of a magnetic bias with both perpendicular and parallel components with respect to the RF magnetic field can be advantageous in terms of frequency tuning range and quality factor. This biasing method was initially suggested by [10]. Moreover, it was shown that doubling the resonance frequency of a measurement cavity is in principal possible by changing a perpendicular magnetic bias field, albeit a radiating open gap degraded the measured $Q$. In [9], it was demonstrated with another ferrite loaded resonant cavity that high quality factors of up to 5000 can be obtained, but in that case, the frequency shift was limited due to a ferrite filling factor of about $25 \%$. The relative permeability for different bias fields for toroidal samples [11] was measured to have suitable input for modeling the relative permeability in simulation programs. Here, the relative permeability and the quality factor of the ferrite material G-510 are investigated with a measurement setup within the frequency range of interest in more detail compared to [12]. In the following, the modeling of the measurement setup in an electromagnetic field simulation program [13] and the comparison of the results with the measurements are presented. Based on this, an electromagnetic design of an accelerating cavity with a frequency sweep of 18 to $40 \mathrm{MHz}$ was made.

\section{Ferrite Loaded Test Setup}

For the accelerating cavity, toroidal cores of the ferrite G-510 of $350 \mathrm{~mm}$ outer and $200 \mathrm{~mm}$ inner diameter consisting of five segments were the largest size currently available. The five segments were attached under mechanical stress to the top of an $3.3 \mathrm{~mm}$ thick $\mathrm{Al} 995$ toroidal carrier disc with the two component epoxy glue Stycast 2850 FT to insure that the azimuthal 


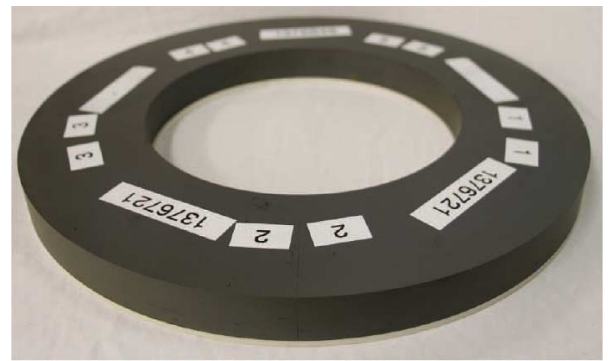

Fig. 1. G-510 ferrite ring composed of five segments $(350 \mathrm{~mm}$ outer, $200 \mathrm{~mm}$ inner diameter and $25.4 \mathrm{~mm}$ height), glued on Al 995 toroidal carrier disc.

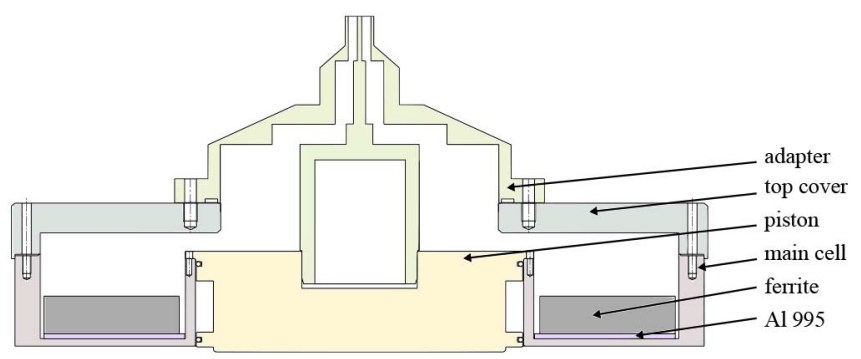

Fig. 2. 1-port reflection measurement setup.

air gaps are small, see Fig. 1. Al 995 provides high thermal conductivity of $29.3 \mathrm{~W} /(\mathrm{m} \cdot \mathrm{K})$, a dielectric loss factor of $\tan \delta_{\mathrm{e}}=2.9 \cdot 10^{-4}$ at $10 \mathrm{MHz}$ and tight fabrication tolerances. $\mathrm{BeO}$ cooling discs were used in earlier works but due to its toxicity during machining and scrapping we try to avoid their use. However, $A l N$ could be an alternative carrier in case an higher thermal conductivity compared to $A l 995$ is required.

\section{A. l-Port Reflection Measurement}

The measurement setup consists of a main cell, a top cover and a piston all made of aluminum. An adapter is connected to the top cover and piston to provide the transition to a $50 \Omega$ type $\mathrm{N}$ connector, see Fig. 2.

The sample holder is filled with one ferrite ring, and the reflection coefficient $S_{11}$ is measured with a Vector Network Analyzer in the range of 1 to $100 \mathrm{MHz}$. For known $\varepsilon^{\prime}=14.3$ and $\tan \delta_{\mathrm{e}}=2 \cdot 10^{-4}$, given by the manufacturer $\mu^{\prime}$ and $\mu^{\prime \prime}$ can be determined from the results of the 1-port reflection measurement. This is described in greater detail in [11] for much smaller ferrite toroids of $70 \mathrm{~mm}$ inner and $127 \mathrm{~mm}$ outer diameter and using a different sample holder and bias system.

The measurement setup is placed within the aperture of an $\mathrm{H}$-dipole to apply a magnetic bias field which is perpendicular to the RF magnetic field, see Fig. 3. The applied magnetic field is measured with a three-axis hall teslameter (THM 7025 from Metrolab Technology SA). Before performing a measurement cycle, the ferrite material is magnetized with a bias field of 300 $\mathrm{mT}$ to bring it into the remanent state. The electromagnet is restricted to a minimum magnetic field of $10 \mathrm{mT}$ while the power supply is running. The measurement results of the relative permeability for increasing magnetic bias fields ( 10 to $300 \mathrm{mT}$ ) are illustrated in Fig. 4. The relative permeability of the G-510 ring, consisting of 5 segments, shows a similar behavior compared to the results for much smaller rings published in [11]. The relative

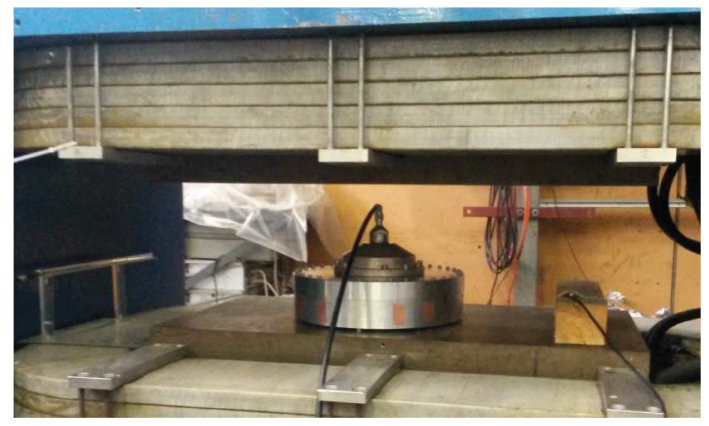

Fig. 3. 1-port reflection measurement setup in aperture of $\mathrm{H}$-dipole magnet.
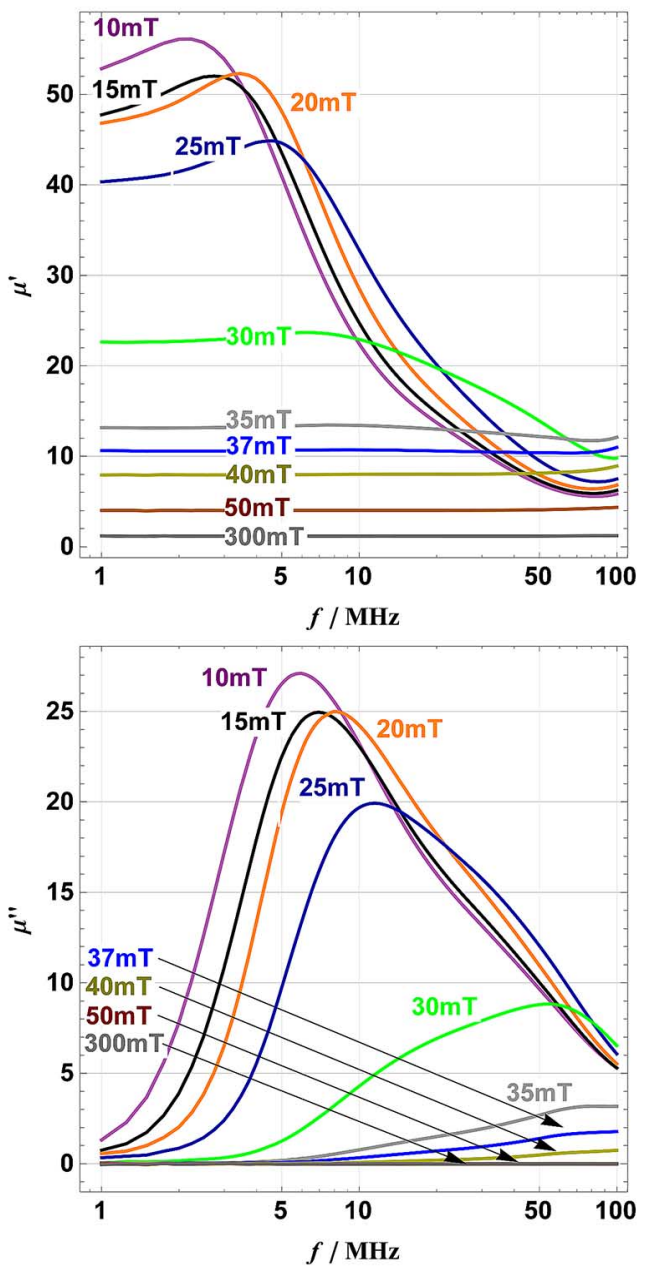

Fig. 4. Complex permeability spectra for increasing magnetic bias field, starting with sample in remanence.

permeability is decreasing and the peak shifts to higher frequencies with increasing magnetic bias field until the real part gets constant and the imaginary part of the relative permeability is coming close to zero.

Fig. 5 shows the relative permeability spectra in detail for a magnetic bias field from 35 to $300 \mathrm{mT}$ within 1 to $50 \mathrm{MHz}$. Above $40 \mathrm{mT}$ bias field, $\mu^{\prime \prime}$ becomes smaller than 0.01 and cannot be calculated accurately because the magnitude of $S_{11}$ approaches unity. For 35,37 and $40 \mathrm{mT}$ the calculated quality factors of the ferrite material from the 1-port measurement are 

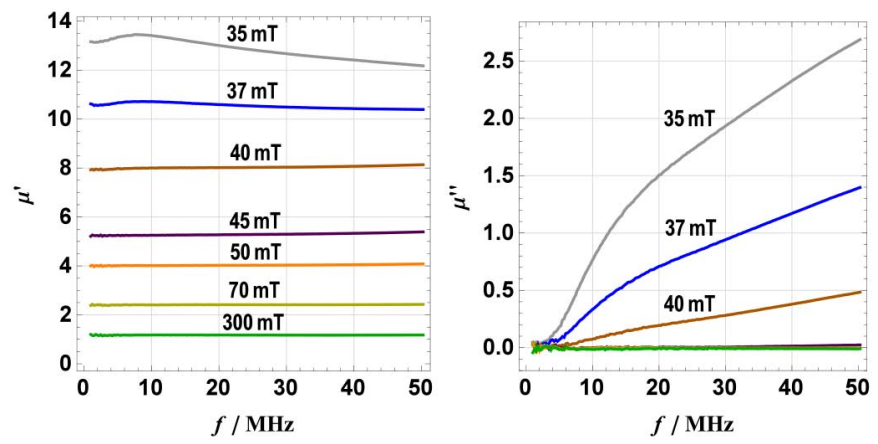

Fig. 5. Complex permeability spectra for $35 \mathrm{mT}$ to $300 \mathrm{mT}$.

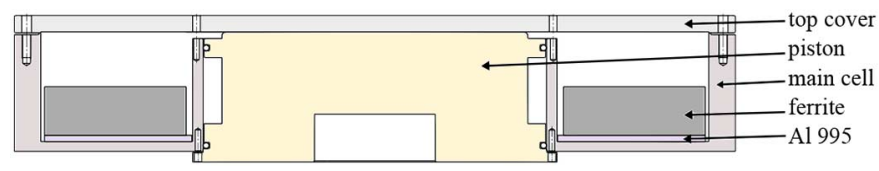

Fig. 6. Resonant measurement setup.

TABLE I

Results of Measurement Setup Filled with 1 and 2 Ferrite Rings

\begin{tabular}{lllllllllll}
\hline \hline \multicolumn{8}{c}{ Results resonant measurement 1 ferrite ring } \\
$\mathrm{B} / \mathrm{mT}$ & - & - & 35 & 37 & 40 & 45 & 50 & 70 & 300 \\
$f_{\text {res }} / \mathrm{MHz}$ & - & - & 18.8 & 20.7 & 23.4 & 27.4 & 30.4 & 36.3 & 43.7 \\
$Q_{\text {total }}$ & - & - & 9 & 16 & 40 & 396 & 1371 & 1274 & 1046 \\
Results 1-Port Reflection Measurement 1 Ferrite Ring \\
$\mu^{\prime}\left(f_{\text {res }}\right)$ & - & - & 13.0 & 10.6 & 8.0 & 5.3 & 4.0 & 2.40 & 1.17 \\
\hline \multicolumn{7}{c}{ Results Resonant Measurement 2 } & Ferrite Rings \\
$\mathrm{B} / \mathrm{mT}$ & 27.5 & 30 & 35 & 40 & 50 & 70 & 110 & 300 & 450 \\
$f_{\text {res }} / \mathrm{MHz}$ & 13.3 & 15.5 & 19.7 & 23.2 & 27.8 & 32.9 & 37.5 & 42.7 & 43.6 \\
$Q_{\text {total }}$ & 10 & 21 & 86 & 745 & 1638 & 1447 & 1280 & 1093 & 1063 \\
Calculated & $\mu^{\prime}\left(f_{\text {res }}\right)$ & from results of numerical simulation & \\
$\mu^{\prime}\left(f_{\text {res }}\right)$ & 13.8 & 9.9 & 6.2 & 4.4 & 3.0 & 2.05 & 1.51 & 1.10 & 1.04 \\
\hline \hline
\end{tabular}

9,15 and 35 respectively, which fits well to the resonant measurement results in the next section.

\section{B. Resonant Measurement}

The test setup is changed in order to perform a resonant measurement as shown in Fig. 6. The piston is turned over and a different top cover is used. A $0.1 \mathrm{~mm}$ Teflon sheet is placed between the top cover and the piston to achieve a capacitive load of up to $1900 \mathrm{pF}$ which reduces the resonance frequency of the empty cavity to $44.7 \mathrm{MHz}$.

In a first step the measurement setup was filled with a single ferrite ring, and in a second run two ferrite rings were placed in the cavity. Both measurement results are compared in Table I. For the measurement with one ferrite ring, no resonance peaks could be detected for magnetic bias fields below $35 \mathrm{mT}$. In that range the losses of the ferrite material are rather high, which was shown in the last section. With increasing magnetic bias field from 35 to $300 \mathrm{mT}$ the resonance frequency shifts from 18.8 to $43.7 \mathrm{MHz}$, due to the decreasing relative permeability of the ferrite material, see Fig. 7. The simulation results which are shown in the figure will be discussed later.

The tuning range of the resonant measurement setup can be increased by placing two ferrite rings within the measurement cavity. The resonance frequency changes from 13.3 to

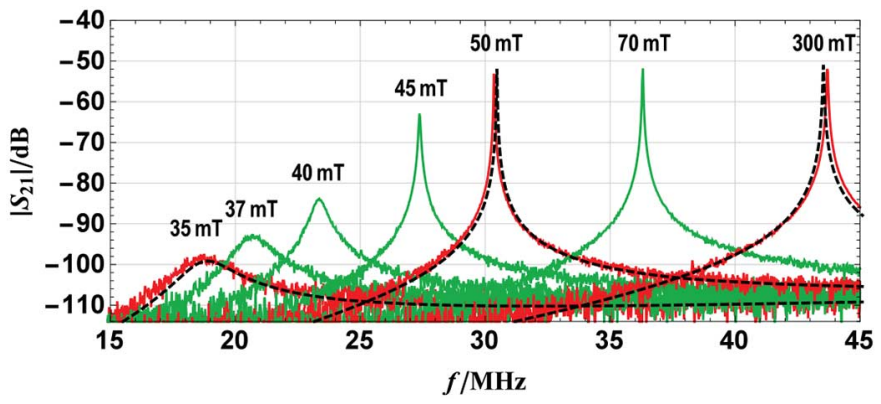

Fig. 7. Measurement results (solid traces) of resonant ferrite filled setup in comparison with driven modal simulation results (dashed traces).

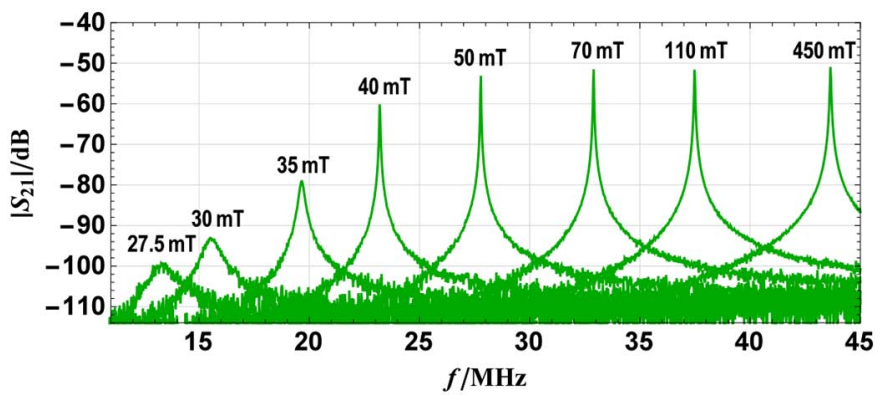

Fig. 8. Measured frequency shift for cavity filled with two ferrite rings.
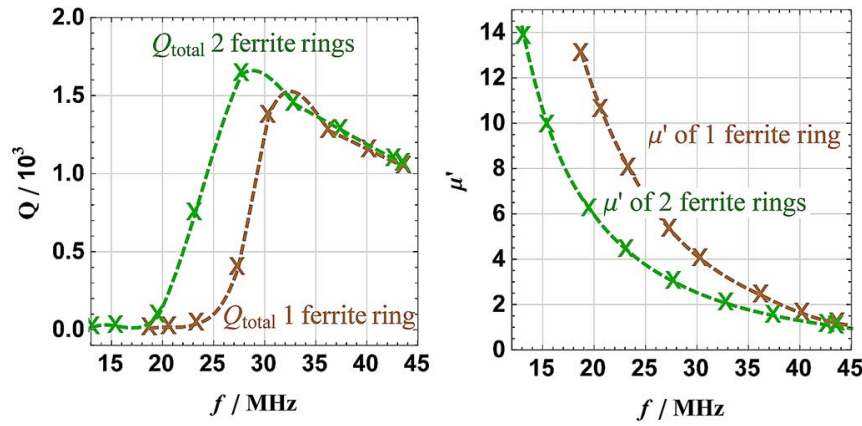

Fig. 9. Left: $Q_{\text {total }}$ of measurement cavity with one and two ferrite rings vs. frequency. Right: $\mu^{\prime}$ of one and two ferrite rings vs. frequency.

43.6 $\mathrm{MHz}$ for a magnetic bias field from 27.5 to $450 \mathrm{mT}$, see Fig. 8. The first resonance peak can be detected at lower magnetic bias field compared to the previous measurement with one ferrite ring. The magnetic field strength within the ferrite ring is dependent on the geometry of the ferrite due to demagnetization fields. A larger ferrite stack leads to a smaller demagnetization factor, hence a smaller external magnetic field is required to achieve the same magnetization of the ferrite material compared to a smaller ferrite stack [14].

The unloaded quality factor can be measured via the $3 \mathrm{~dB}$ bandwidth of the transmission signal as weak couplings are used and monitored. For both setups, the measured quality factor first increases to a maximum which is well above the quality factor of the empty cavity of 1129 and then decreases with increasing bias field. The quality factor of the resonant cavity filled with one and two ferrite rings over the bias field is shown in Fig. 9, left and the related relative permeability over the bias field is illustrated in the right plot. The larger ferrite filling factor of using two ferrite rings leads to a larger overall tuning range and higher 


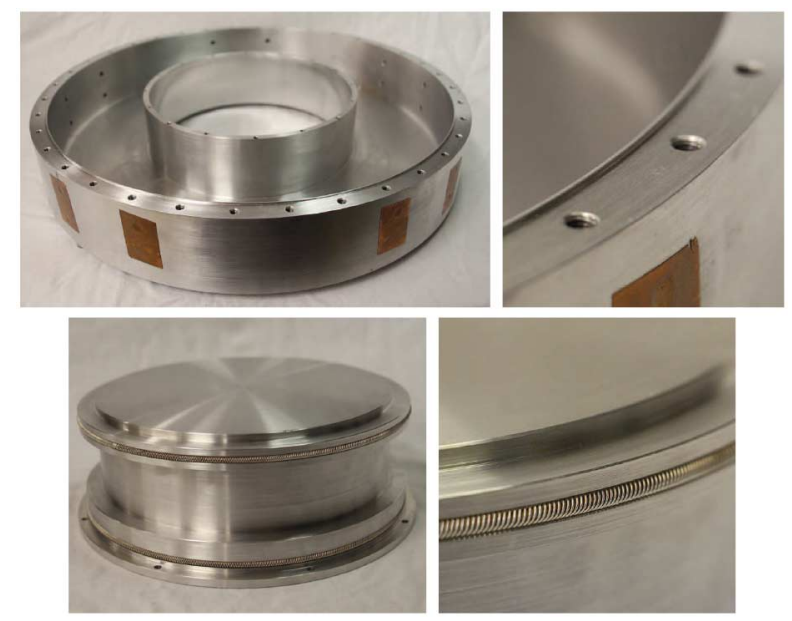

Fig. 10. Top: Picture of RF-contact on main cell. Bottom: Picture of RF-contact on piston.

quality factor. In the next section, the resonant measurement setup is modeled in an electromagnetic field simulation program to investigate this phenomena.

\section{INVESTIGATION OF QUALITY FACTOR WITH NUMERICAL Simulations AND CROSS-CHECK OF Simulation RESUltS}

The resonance frequency of the measurement cavity is sensitive to the capacitive load. Tightening the screws on the cavity lid leads to a bending of the top cover and a local air-gap occurs which results in a deviation of the resonance frequency. The effect of the local air-gap can be compensated in the simulation model by adding an effective air-gap of $60 \mu \mathrm{m}$ to the $0.1 \mathrm{~mm}$ Teflon sheet. This way a matching of simulated and measured resonance frequency could be achieved. For the test setup, aluminum was chosen as cavity material in order to keep the total weight low, while staying with a comparable good electric conductivity. However, the quality factor of the simulation model is about $38 \%$ larger compared to the measurement of the empty cavity. Within the simulation model only ohmic losses were taken into account and contact resistances were neglected. To overcome the known problems of aluminum in terms of RF contact resistance, all contact surfaces were treated with abrasive paper and alcohol before the measurements. Between main cell and top cover a $1 \mathrm{~mm}$ wide edge was integrated to provide a good contact over the full cavity diameter, see Fig. 10, top. In addition, a RF spring contact was inserted on the critical area in between piston and the inner conductor of the main cell as shown in Fig. 10, bottom.

Hence, as a first measure, the surface conductivity of the simulation model is reduced to bring the simulated quality factor in agreement with the measurement. The quality factor of the ferrite loaded measurement cavity can be expressed as:

$$
\frac{1}{Q_{\mathrm{total}}}=\frac{1}{Q_{\Omega}\left(\mu_{\mathrm{eff}}^{\prime}, f\right)}+\frac{1}{Q_{\varepsilon, \mathrm{eff}}}+\frac{1}{Q_{\mu, \mathrm{eff}}} .
$$

where $Q_{\Omega} \sim \mu_{\text {eff }}^{\prime} \sqrt{f}, Q_{\varepsilon, \text { eff }}$ and $Q_{\mu \text {,eff }}$ refer to the ohmic, dielectric and magnetic quality factor, respectively. It should be pointed out that calculating the magnetic quality factor of the ferrite material with Eq. (1) requires the usage of energy scaling
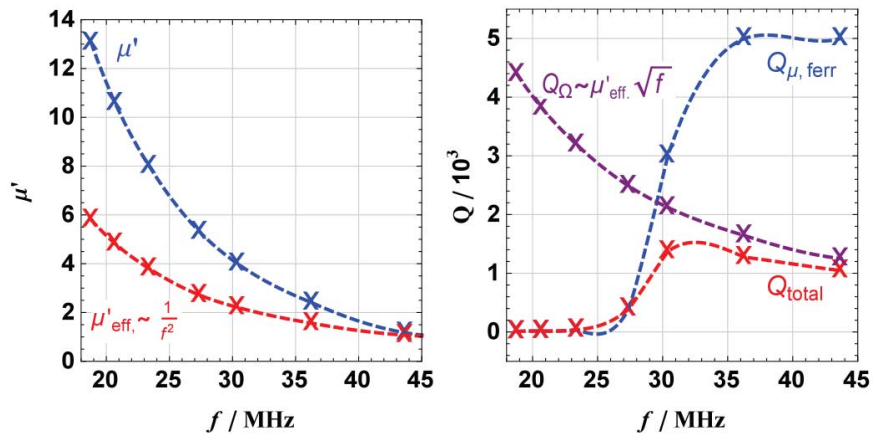

Fig. 11. Left: $\mu^{\prime}$ and $\mu_{\text {eff }}^{\prime}$ vs. $f$. Right: $Q_{\text {total }}, Q_{\mu, \text { ferr }}$ and $Q_{\Omega}$ vs. $f$.

factors, similar to [15]. Furthermore, it has to be considered, that the ohmic quality factor is also dependent on the electromagnetic properties of the filling material i.e., it can increase when the resonator is loaded with high- $Q$ ferrite. In this case, the presence of the ferrite reduces the surface current density on the inner wall for equal amount of stored energy. The magnetic energy stored in the Teflon sheet, the electric energy stored in the ferrite toroid and in the Al 995 carrier disc can be neglected due to the electromagnetic field distribution in the re-entrant cavity. Hence, it is sufficient to take only the ohmic quality factor of the cavity $Q_{\Omega}$, the dielectric quality factor of the Teflon $Q_{\varepsilon}$, Teflon and the magnetic quality factor of the ferrite material $Q_{\mu \text {,ferr }}$ into account to express the total quality factor. Both $Q_{\varepsilon}$, Teflon and $Q_{\mu}$,ferrite need to be multiplied by an energy filling factor to obtain the dielectric and magnetic $Q$ of the cavity according to:

$$
\begin{aligned}
Q_{\varepsilon, \mathrm{eff}} & =\frac{W_{\mathrm{el}, \text { total }}}{W_{\mathrm{el}, \text { Teflon }}} Q_{\varepsilon, \text { Teflon }} \\
\text { and } Q_{\mu, \mathrm{eff}} & =\frac{W_{\mathrm{mag}, \text { total }}}{W_{\mathrm{mag}, \mathrm{ferr}}} Q_{\mu, \mathrm{ferr}}
\end{aligned}
$$

In addition to the investigation of the quality factor of the ferrite material, the numerical simulation is also used to verify the resonant measurement results. The relative permeability of the ferrite is modeled based on the 1-port reflection measurement results for a specific magnetic bias field. The results of an Eigenmode simulation of the resonant cavity are compared with the resonant measurement in Table II. The relative error between measured and simulated resonance frequency is smaller than $1.5 \%$. Moreover, the results of a driven modal simulation compared to the measurement results are shown in Fig. 7 . Besides the resonance frequencies, the peak values of the transmission signal also show good agreement and both deviate by less than $1.5 \%$ from the measurement results. The effective relative permeability of the cavity $\mu_{\text {eff }}^{\prime}$ can be calculated from the simulation results by multiplying the relative permeability of the ferrite $\mu^{\prime}$ with a filling factor, both are compared in Fig. 11, left. This shows quite well that a higher ferrite filling factor increases the effective permeability of the cavity and increases the tuning range, as it is the case for using two ferrite rings.

$Q_{\mu, \text { ferrite }}$ is adjusted as input for the numerical simulation in order to bring the quality factor of the simulation of the ferrite loaded cavity in agreement with the measurement (relative error $<5 \%$ ). The simulation results have been verified by Eq. (1) and 
TABLE II

Resonant Measurement Compared to Eigenmode Simulation RESUlts WITH RELATIVE PERMEABILITY INPUT FROM 1-PORT REFLECTION MEASUREMENT OF 1 FERRITE RING

\begin{tabular}{lccccccc}
\hline \hline $\mathrm{B} / \mathrm{mT}$ & 35 & 37 & 40 & 45 & 50 & 70 & 300 \\
\hline \multicolumn{7}{c}{ Results resonant measurement } \\
$f_{\text {res }} / \mathrm{MHz}$ & 18.8 & 20.7 & 23.4 & 27.4 & 30.4 & 36.3 & 43.7 \\
$Q_{\text {total }}$ & 9 & 16 & 40 & 396 & 1371 & 1274 & 1046 \\
\hline Input simulation: $\mu^{\prime}\left(f_{\text {res }}\right)$ & from measurement, adapted $Q_{\mu, \text { ferr }}$ \\
$\mu^{\prime}\left(f_{\text {res }}\right)$ & 13.0 & 10.6 & 8.00 & 5.30 & 4.00 & 2.40 & 1.17 \\
$1 / Q_{\mu, \text { ferr }}$ & $1 / 8$ & $1 / 15$ & $1 / 35$ & $1 / 396$ & $1 / 3003$ & $1 / 5000$ & $1 / 5000$ \\
\hline \multicolumn{7}{c}{ Results simulation } \\
$f_{\text {res }} / \mathrm{MHz}$ & 18.6 & 20.5 & 23.1 & 27.3 & 30.3 & 35.9 & 43.3 \\
$\delta_{\text {fres }} / \%$ & 1.1 & 1.0 & 1.3 & 0.4 & 0.3 & 1.1 & 0.9 \\
\hline \hline
\end{tabular}

(2). The different $Q$ factors are compared in Fig. 11, right. It can be seen that $Q_{\mu \text {,ferr }}$ is small for low magnetic bias fields, but increases significantly up to a maximum of 5000 once magnetic saturation is reached. $Q_{\Omega}$ decreases with increasing frequency and can be considered to be proportional to the effective relative permeability of the cavity and the square root of the frequency with reasonable accuracy. The total quality factor of the cavity is first dominated by the magnetic quality factor of the ferrite for low frequencies. Once the magnetic $Q$ of the ferrite is greater than the ohmic $Q, Q_{\text {total }}$ reaches a maximum, which is above the $Q_{\text {total }}$ of the empty cavity and then decreases with increasing frequency. However, here it is shown that a maximum $Q_{\mu, \text { ferr }}$ of 5000 can be achieved, which agrees well with Hutcheon's results [16] of a $Q_{\mu \text {,ferr }}$ of $500-5000$ for a relative permeability variation of $4-1.25$ and also with the measured $Q$ of 5000 for the ferrite loaded copper cavity presented in [9].

\section{Preliminary Design of Ferrite LoAded ACCELERATING CAVITY}

In order to investigate the suitability of the G-510 material as a perpendicular biased tuning ferrite, a simplified ferrite loaded accelerating cavity was designed. The power needed to achieve $1 \mathrm{kV}$ accelerating voltage was calculated by means of an Eigenvalue simulation. In a preliminary design lattice, the maximum space foreseen per cavity was given with $1125 \mathrm{~mm}$. Furthermore, an accelerating gap of $50 \mathrm{~mm}$, a beam pipe of $2 \mathrm{~mm}$ thickness and $130 \mathrm{~mm}$ diameter are assumed. A ferrite stack of 20 rings with $25.4 \mathrm{~mm}$ thickness each was modeled by using the data taken from the 1-port reflection measurement with 1 ferrite ring. By using ferrite rings of $350 \mathrm{~mm}$ outer diameter, the required resonance frequency range of 18 to $40 \mathrm{MHz}$ could not be achieved. As a first measure, larger ferrite rings rings of $200 \mathrm{~mm}$ inner and $600 \mathrm{~mm}$ outer diameter were used for the preliminary design shown in Fig. 12.

The two operation points of the accelerating cavity are simulated with an Eigenmode solver and the results are listed in Table III. For the low frequency operation point, a relative permeability of 8 and a corresponding $Q_{\mu \text {,ferrite }}$ of 35 will lead to a resonance frequency of $17.6 \mathrm{MHz}$ and a $Q_{0}$ of 37 . Note, that $Q_{\mu \text {,ferrite }}$ is the magnetic $Q$ of the ferrite and has to be scaled with an energy filling factor to obtain the magnetic $Q$ of the cavity according to Eq. (1) and (2). This is the reason why $Q_{0}$ is larger compared to $Q_{\mu, \text { ferrite }}$ in case of the low frequency operation point. With a $R / Q$ of $213 \Omega$ calculated from the simulation

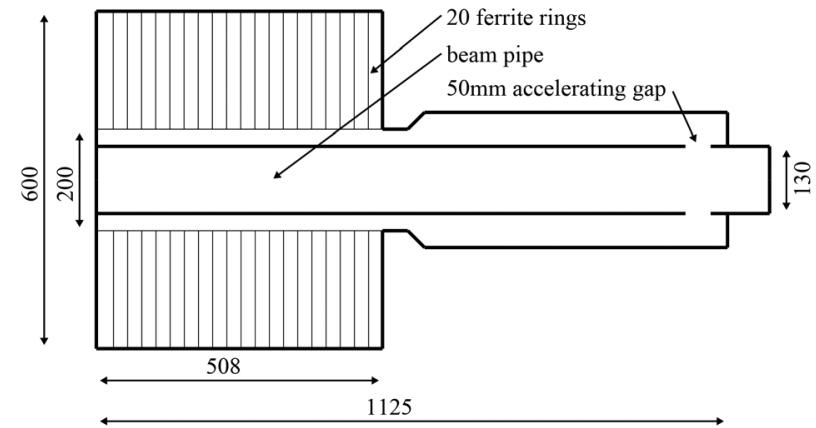

Fig. 12. Preliminary design of accelerating cavity, all dimensions in $\mathrm{mm}$.

TABLE III

Simulation Results of FLC and Estimated Power NeEdS for $1 \mathrm{KV}$ ACCELERATing VOltage

\begin{tabular}{ll|llll}
\hline \hline \multicolumn{2}{c}{ Input Simulation } & \multicolumn{5}{c}{ Results Simulation } \\
$\mu^{\prime}$ & $Q_{\mu, \text { ferr }}$ & $f_{\text {res }} / \mathrm{MHz}$ & $Q_{0}$ & $\frac{R}{Q} / \Omega$ & $P / \mathrm{W}$ \\
\hline 8 & 35 & 17.6 & 37 & 213 & 63.3 \\
1.17 & 5000 & 40.9 & 4683 & 108 & 1 \\
\hline \hline
\end{tabular}

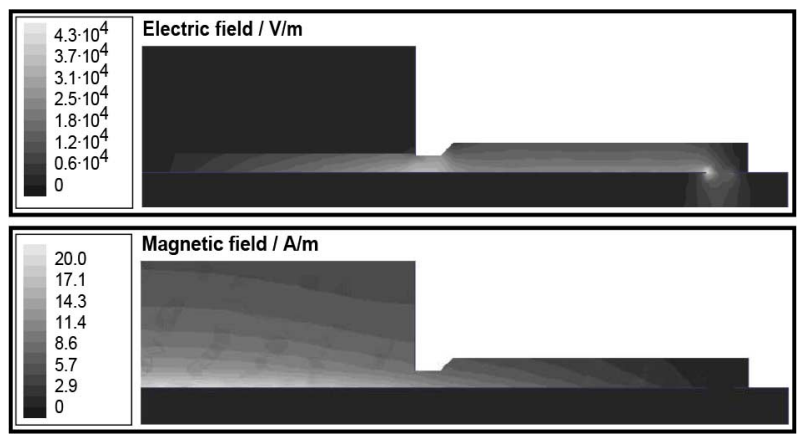

Fig. 13. Preliminary design of accelerating cavity. Top: electric RF field distribution, bottom: magnetic RF field distribution for $\mu^{\prime}=8$. Picture taken from [13].

results, $63.3 \mathrm{~W}$ power are required to achieve an accelerating voltage of $1 \mathrm{kV}$. The RF field distribution is shown in Fig. 13.

For the high frequency operation point the relative permeability of the ferrite is set to 1.17 , which leads to a resonance frequency of $40.9 \mathrm{MHz}$. The unloaded quality factor of the ferrite loaded cavity will be 4683 , hence with a $R / Q$ of $108 \Omega$, $1 \mathrm{~W}$ power will be needed per $1 \mathrm{kV}$ accelerating voltage. By considering the aforementioned study, for example, an accelerating voltage of $62.5 \mathrm{kV}$ would be needed at $40 \mathrm{MHz}$ and $8.3 \mathrm{kV}$ would be required at $18 \mathrm{MHz}$; in both cases about $4 \mathrm{~kW}$ of RF power would be needed. This rough estimate shows that the required frequency range of 18 to $40 \mathrm{MHz}$ could be covered with reasonable power consumption.

\section{CONCLUSION}

A test setup to characterized the complex permeability of full scale G-510 ferrite rings as a function of the magnetic bias field was presented. The resonance frequency shift based on a changing magnetic bias field was investigated with a resonant measurement and the quality factor of the ferrite material was determined. It was possible to reproduce the results of the resonant measurement with a numerical simulation by modeling the 
ferrite with the measured relative permeability. Based on the results obtained, a preliminary design of a ferrite filled accelerating cavity to achieve a frequency sweep of 18 to $40 \mathrm{MHz}$ was studied.

\section{OUTLOOK}

For measurement and simulation an homogenous relative permeability distribution within the ferrite volume was assumed. But in fact, due to demagnetization fields, an inhomogeneous distribution of the relative permeability is obtained. As a next step, this influence should be analyzed. Furthermore, the magnetic losses of the ferrite are thought to rise with increasing RF power and this has to be investigated. Moreover, the preliminary design has to be further elaborated taking into account the maximal available size of ferrite rings produced in industry.

\section{ACKNOWLEDGMENT}

The authors would like to thank E. Jensen and C. Rossi from CERN for their support and Prof. H. Klingbeil from TU Darmstadt for valuable discussions.

\section{REFERENCES}

[1] L. M. Earley et al., "A high-Q ferrite-tuned cavity," IEEE Trans. Nucl. Sci., vol. NS-30, no. 4, pp. 3460-3462, 1983.
[2] P. Coleman et al., "Status of the SSC LEB RF cavity," in Proc. PAC, 1993, pp. 824-826.

[3] R. L. Poirier et al., "AC bias operation of the perpendicular biased ferrite tuned cavity for the TRIUMF KAON factory boostesr synchrotron," in Proc. PAC, 1991, pp. 2943-2945.

[4] G. Romanov et al., "Perpendicular biased ferrite tuned cavities for the Fermilab booster," in Proc. IPAC, 2014, THPRI061.

[5] C. Y. Tan et al., "A perpendicular biased 2nd harmonic cavity for the Fermilab booster," in IPAC, 2015, WEPTY037.

[6] C. Vollinger et al., "Accurate measurement of ferrite garnets to be used for fast-tuned ferrite loaded cavities in the range of $20-40 \mathrm{MHz}$," in Proc. IPAC, 2012, THPPC020.

[7] Trans-Tech, Inc. [Online]. Available: http://www.trans-techinc.com/

[8] C. Vollinger et al., "The effect of 2-directional magnetic biasing used for tuning of a ferrite-loaded re-entrant cavity," IEEE Trans. Nucl. Sci. vol. 60 , no. 3, pp. 2170-2174, Jun. 2013.

[9] C. Vollinger et al., "Enlargement of tuning range in a ferrite-tuned cavity through superposed orthogonal and parallel magnetic bias," in Proc. IPAC, 2013, WEPFI054.

[10] W. R. Smythe et al., "Reducing ferrite tuner power loss by bias field rotation," IEEE Trans. Nucl. Sci., vol. NS-30, no. 4, pp. 273-275, 1983.

[11] J. Eberhardt et al., "Ferrite material characterization in a static bias field for the design of a tunable cavity," in Proc. IPAC, 2014, THPRI053.

[12] J. Eberhardt et al. "Preliminary design of a perpendicular biased ferrite loaded accelerating cavity," in Proc. IPAC, 2015, WEPHA024.

[13] ANSYS HFSS, Release 15.0, ANSYS, Inc. [Online]. Available: http:// www.ansys.com/

[14] D. M. Pozar, "Theory and design of ferrimagnetic components," in $\mathrm{Mi}$ crowave Engineering, 3rd ed. Hoboken, NJ, USA: Wiley, 2012, ch. 9 , sec. 1 , pp. $451-454$.

[15] W. R. Smythe et al., "RF cavities with transversely biased ferrite tuning," IEEE Trans. Nucl. Sci., vol. NS-32, no. 5, pp. 2951-2953, 1985

[16] R. M. Hutcheon, "A perpendicular-biased ferrite tuner for the $52 \mathrm{MHz}$ petra II cavities," in Proc. PAC, 1987. 